\title{
STOCHASTIC INTEGRAL INEQUALITIES WITH APPLICATIONS
}

\author{
MENG Wu AND NAN-JING HUANG
}

\begin{abstract}
In this paper, we study some new stochastic inequalities involving the Itô integral and give some estimates for the solutions of controlled stochastic differential equations. As applications, we utilize the stochastic integral inequalities presented in this paper to show an existence theorem of the solution for a class of stochastic differential equations and to give necessary conditions that make the solution for a class of stochastic differential equations be a diffusion process.
\end{abstract}

Mathematics subject classification (2010): 26D10, 26D20, 60H05, 60H35.

Keywords and phrases: Stochastic integral inequality, Itô integral, stochastic differential equation, diffusion process.

\section{REFERENCES}

[1] K. Amano, A Stochastic Gronwall Inequality and Its Applications, J. Inequal. Pure Appl. Math., 6(1) (2005), Art. 17.

[2] A. JoUlin, On maximal inequalities for stable stochastic integrals, Potential Anal. 26 (2007), 57-78.

[3] I. Karatzas And S. E. Shreve, Brownian Motion and Stochastic Calculus, Springer-Verlag, New York, 1988.

[4] N. El Karouri, S. Peng And M. C. QueneZ, Backward stochastic differential equation in finance, Mathematical Finance, 7 (1997), 1-71.

[5] H. H. KuO, Introduction to Stochastic Integration, Spriger-Verlag, New York, 2006.

[6] J. Ma AND J. M. Yong, Forward-Backward Stochastic Differential Equations and Their Applicaitons, Springer-Verlag, Berlin, 1999.

[7] C. Mueller And A. Stan, A Heisenberg inequality for stochastic integrals, J. Theoret. Probab. 18 (2005), 291-315.

[8] B. ØKSENDAL, Stochastic Differential Equation, An Introduction with Applications, 6th Edition, Springer-Verlag, Berlin, 2003.

[9] P. H. THEOdORE AND H. Christian (EDS.), Stochastic Inequalities and their Applications, Contemporary Mathematics, 234, American Mathematical Society, Providence, RI, 1999.

[10] Y. L. TONG, Relationship between stochastic inequalities and some classical mathematical inequalities, J. Inequal. Appl. 1(1997), 85-98.

[11] L. T. YAN, Some ratio inequalities for iterated stochastic integrals, Math. Nachr. 259 (2003), 84-98.

[12] L. T. YAN, Two inequalities for iterated stochastic integrals, Arch. Math. (Basel) 82 (2004), 377-384. 